Itinéraires Itinéraires

Littérature, textes, cultures

\title{
Banlieue et dystopie en littérature urbaine : les cas de Zone cinglée et de René
}

Banlieue and Dystopia in Urban Literature: The Cases of Zone cinglée and René

\section{Stève Puig}

\section{OpenEdition}

\section{Journals}

Édition électronique

URL : http://journals.openedition.org/itineraires/3546

DOI : 10.4000/itineraires.3546

ISSN : 2427-920X

Éditeur

Pléiade

Référence électronique

Stève Puig, "Banlieue et dystopie en littérature urbaine : les cas de Zone cinglée et de René », Itinéraires [En ligne], 2016-3 | 2017, mis en ligne le 15 juillet 2017, consulté le 19 avril 2019. URL : http:// journals.openedition.org/itineraires/3546 ; DOI : 10.4000/itineraires.3546

Ce document a été généré automatiquement le 19 avril 2019

\section{$\circledast \oplus \Theta \Theta$}

Itinéraires est mis à disposition selon les termes de la licence Creative Commons Attribution - Pas d'Utilisation Commerciale - Pas de Modification 4.0 International. 


\title{
Banlieue et dystopie en littérature urbaine : les cas de Zone cinglée et de René
}

\author{
Banlieue and Dystopia in Urban Literature: The Cases of Zone cinglée and
}

René

Stève Puig

1 La littérature urbaine, mouvance littéraire émergeant peu après les émeutes de 2005 (qualifiées elles-mêmes d'émeutes urbaines), offre des représentations de la banlieue variées, qui oscillent entre le constat de difficultés socio-économiques et la fierté d'appartenir à un espace pourtant fortement stigmatisé. Le premier roman de Kaoutar Harchi, Zone cinglée, ainsi que le deuxième roman du rappeur Disiz, intitulé René, offrent des images originales de la banlieue, qui évoquent un genre de plus en plus célèbre en littérature et à la télévision: celui de la dystopie. En tant que genre, la dystopie s'apparente à une contre-utopie, c'est-à-dire à un récit dans lequel la société est devenue victime de ses propres maux, souvent liés à la surabondance des nouvelles technologies, à des questions politiques, ou encore à des problèmes résultant de l'urbanisme (ce qui concerne la littérature urbaine).

Dans le cas de Zone cinglée, paru en 2009 aux éditions Sarbacane, les femmes d'une banlieue s'unissent pour habiter une partie de la ville isolée et défendre leur cause : créer une nouvelle génération d'enfants élevés exclusivement par des femmes, s'opposant ainsi à l'ancienne, ravagée par la drogue et les crimes. Les différentes zones d'habitation du roman évoquent un urbanisme cauchemardesque dans lequel il devient quasiment impossible de passer d'une zone à l'autre. Dans René, paru chez Denoël en 2012, Disiz prend comme décor une France du futur tombée aux mains du Front national et la vie dans une banlieue délabrée, morcelée, et complètement coupée de la capitale, avec en fond, la menace d'une guerre civile.

3 En insistant sur les dangers d'un urbanisme mal géré et sur les problèmes liés à la ségrégation urbaine, ces deux romans offrent donc une vision des banlieues pouvant être 
qualifiée d'apocalyptique (étymologiquement, le mot signifie aussi « révélation»). Plutôt que de se limiter à des récits de type sociologique, les deux écrivains préfèrent ancrer leur roman dans le cadre de la dystopie qui, en tant que genre, leur permet indirectement de faire un constat alarmant sur les conditions de vie dans certaines banlieues tout en alertant le lecteur d'éventuels dérapages de la société française actuelle dans son ensemble.

Le concept de dystopie existe plutôt dans un contexte anglo-saxon; le terme lui-même venant de l'anglais «dystopia ». Rappelons que le mot « utopia », popularisé par Thomas More, contient une signification double: l'utopie est à la fois un endroit idéal et étymologiquement un lieu qui n'existe pas. Le préfixe dys- quant à lui est emprunté au grec dus et indique la négation, ou la malformation (que l'on retrouve dans le mot « dysfonctionnement » par exemple). Une dystopie est donc une utopie qui tourne mal, et qui sert en général à attirer l'attention du lecteur sur des problèmes de société actuels en forçant le trait. En France, Valérie Stiénon estime que les premières dystopies datent du début du XIX ${ }^{e}$ siècle ${ }^{1}$. Sans vouloir refaire une généalogie des dystopies françaises, on peut citer parmi les plus célèbres celle de Pierre Boule (La Planète des singes, 1963) et, plus récemment Amélie Nothomb (Acide sulfurique, 2005) imagine une téléréalité extrême dans laquelle les personnages, kidnappés dans la rue, finissent dans un camp de travail forcé, avec, pour les perdants, la menace d'une exécution en direct.

\section{Zone cinglée : l'écriture du chaos urbain}

5 Dans Zone cinglée, Kaoutar Harchi imagine une ville dans laquelle les femmes (les Mères) créent une communauté exclusivement féminine appelée la Cause, qui vit en retrait dans une partie sombre de la ville. Cette ville est divisée en plusieurs territoires, avec, entre autres, la zone nord, où règnent une misère et un chaos extrêmes, la zone sud, celle des Mères, la zone appelée l'Antre, dans laquelle le personnage principal erre pour se retrouver en présence d'autres hommes, et la ville-centre, refuge de jeunes femmes qui ont voulu échapper à la Cause ainsi qu'à la misère de la zone nord. La distinction entre zone nord et zone sud est donc importante dans le roman, et les diverses parties de la ville indiquent la volonté de l'écrivaine d'évoquer les clivages socio-économiques qui existent dans bon nombre de grandes villes françaises, notamment entre le sud et le nord, ou, comme c'est le cas de certaines villes, entre l'est et l'ouest. Tâarouk, le personnage principal du roman et le narrateur, se définit au début du roman comme étant un produit de cette cité isolée du reste du monde, plongée dans l'obscurité, et presque exclusivement masculine :

Mon nom est Tâarouk. J'ai 26 ans. J'habite au seizième étage d'un immeuble sans ascenseur. Les architectes ont construit des tours très hautes pour nous cacher dans le ciel [...]. Je vis dans une zone hachurée. Striée par ces barres verticales qui découpent l'espace en fines bandelettes de terre. De béton. Ici l'ombre alterne avec l'ombre. Des gens habitent. Je veux dire, vivent. Et meurent dans ces territoires étroits. La cité est un cercle dans le vide, qu'une bande rugueuse rattache au monde. (Harchi 2009 : 14)

6 Cet extrait ancre le roman dans la mouvance urbaine (Puig 2011) dont les caractéristiques sont, entre autres, un paratexte évoquant clairement des thèmes liés à l'urbanisme, notamment la couverture qui arbore des logements du type "grands ensembles" symbolisant les périphéries françaises, et des descriptions des logements en banlieue, avec parfois l'évocation de tours très hautes (comme dans la nouvelle «Détours » dans 
Chroniques d'une société annoncée du collectif Qui Fait la France ?). Ce passage évoque également l'isolement de cette zone et le sentiment d'enfermement très répandu lui aussi dans la littérature urbaine (voir Boumkoeur de Rachid Djaïdani dans lequel les personnages sont cachés dans une sorte de bunker). Ce thème est d'ailleurs présent dans l'incipit même, puisque Kaoutar Harchi utilise un passage de Nedjma, le roman de Kateb Yacine : «Lakhdar s'est échappé de sa cellule ", évoquant la capture du personnage. Le titre lui-même Zone cinglée qui juxtapose un terme géographique et un état, celui de la folie, contribue à évoquer à travers la personnification une sorte d'asile en plein air, mais isolé, ce qui renvoie à un autre thème récurrent dans la littérature et le cinéma urbains : celui d'être « enfermés dehors » (pour utiliser la fameuse expression présente dans le film La Haine par exemple). Cette expression, qui paraît paradoxale au premier abord, a du sens dans la mesure où les jeunes de banlieues, souvent pour éviter des appartements exigus, préfèrent se retrouver en bas des tours, mais d'un autre côté, se sentent prisonniers de leurs cités.

7 Le terme de «zone » utilisé pour décrire la partie de la ville où vivent les Mères, réfère bien entendu à un mot ayant souvent une connotation péjorative, dont est tiré le mot « zonard » par exemple ou « zonier » que l'on trouve parfois dans la littérature, décrivant un habitant d'une banlieue perçu comme étant peu fréquentable. La situation dans le roman est celle d'une lutte de la part des Mères qui veulent rallier toutes les femmes à leur cause. L'emploi du mot «zone » dans ce contexte renforce donc l'impression d'une guerre urbaine (on parle dans un contexte militaire de zone libre, zone occupée, zone militarisée ou démilitarisée). En urbanisme, la zone est un faubourg caractérisé par un habitat misérable ; une banlieue industrielle, mal aménagée, d'une grande agglomération urbaine. Le terme évoque les acronymes ZUP et ZEP qui ont malheureusement contribué à la mauvaise réputation de certains quartiers ou de certaines villes et il évoque une époque où les alentours de la capitale n'étaient pas forcément très délimités dans l'imaginaire parisien. Par exemple, on trouve chez Louis-Ferdinand Céline l'évocation de la zone au-delà des " fortifs »: " cette espèce de village qui n'arrive jamais à se dégager tout à fait de la boue, coincé dans les ordures » (Céline 1932: 333). Cette connotation péjorative est d'ailleurs restée dans le parler familier (lorsque l'on dit «c'est la zone » pour évoquer une situation chaotique).

8 C'est bien une impression de chaos ou d'apocalypse qui règne dans Zone cinglée où l'urbanisme et les contraintes spatiales ont rendu folles les Mères qui ont décidé de s'enfermer de leur propre gré dans un recoin isolé de la ville. Le thème de l'enfermement, qui contribue donc à l'atmosphère étouffante quasiment claustrophobe du roman, est lié à la question du genre (gender), puisqu'apparaît dans le livre une division en quatre zones : pour les hommes, la zone nord, misérable, où vit le narrateur ; l'antre pour les homosexuels, dans laquelle il erre; pour les femmes, la zone sud pour les Mères ; la villecentre, pour les jeunes filles ayant voulu s'émanciper de la domination masculine. La mère de Tâarouk peut être perçue comme une victime de cette domination des hommes qui régnait sur la ville avant que les femmes ne s'insurgent. En effet, le jeune homme affirme que sa mère, n'ayant pas pu vivre de manière libre, est morte à petit feu :

Pour Maman, chaque jour a été un pas de plus vers l'enfermement. Elle a résisté longtemps à l'inéluctable avancée des murs autour d'elle, c'était Bâ qui les poussait de toutes ses forces. Fatalement, l'espace s'est réduit. La lumière a baissé. Jusqu'à ce que maman devienne un point dans le noir. Elle est morte un début de printemps, d'épuisement. (Harchi $2009: 22)$ 
Les Mères qui habitent la « zone cinglée » sont décrites tantôt comme des monstres tantôt comme des folles, mais leur description font qu'elles sont apparentées à des amazones ou à des harpies à cause de leur rapidité (37). À travers ces descriptions, elles font basculer le roman tantôt vers la science-fiction, donc plutôt vers le futur, tantôt vers la mythologie, tantôt vers le fantastique, ce qui pose la question du genre du roman.

\section{Un texte hybride}

10 Du point de vue de la structure, le roman est divisé en cinq actes, évoquant une tragédie classique inspirée par la mythologie grecque. D'autres éléments contribuent à faire de Zone cinglée une tragédie moderne ou peut-être postmoderne, comme le nom de l'ami de Tâarouk, Izare, qui évoque Icare, un symbole de liberté car dans la mythologie grecque, Icare, fils de Dédale, voulait s'échapper du labyrinthe avec des ailes de cire créées par son père. Un des personnages s'appelle Abel, une référence religieuse (au frère de Caïn, tué par ce dernier) qui contribue également à donner au roman un aspect intemporel, mythique. D'autre part, certains gardes sont qualifiés de Cerbères, ce qui renvoie encore à la mythologie, et accentue le caractère infernal de la ville (Cerbère étant le chien monstrueux à trois têtes, gardien de l'entrée des Enfers).

11 Le roman est donc un mélange de références au passé, à la mythologie, et à la sciencefiction que l'on a parfois tendance à aborder d'un point de vue très futuriste, alors que certaines œuvres de science-fiction effectuent au contraire un retour vers le passé, voire à un âge quasi-primitif. Évelyne Bornier voit dans Zone Cinglée une sorte d'Apocalypse Now "made in banlieue ", qui nous éloigne des clichés habituellement associés à la banlieue : «Zone cinglée dérange, déboulonne les mythes de la cité qu'il dénonce comme éculés, et nous offre une vision apocalyptique, choquante, et sans fioritures de cette dernière ; celle des derniers jours d'une banlieue en mode auto-destructeur» (Bornier 2012: 378). On peut aussi voir Zone cinglée comme un roman fantastique, qui nous fait hésiter entre réalité et science-fiction (pour Todorov, le fantastique naît justement de cette hésitation entre réalisme et surnaturel ou merveilleux). Cette frontière entre réalisme et surnaturel représente alors une autre frontière dans le roman. Le portrait des Mères par exemple illustre bien ce propos puisque leur description tient parfois du réalisme, parfois de la mythologie (elles sont décrites comme des harpies avec une force quasi surnaturelle) :

Je comprends que malgré la rage et la peine, elles sont restées des femmes. Elles ont un buste, des bras et des jambes d'allumettes. C'est à l'intérieur que tout est bétonné. Le ciment des trottoirs et des tours s'est infiltré en elles comme l'eau dans une fissure. Elles en sont pleines. Leur corps ne ressent plus rien. C'est fini. (Harchi 2009 : 89-90)

12 Le lecteur peut lire cette description soit comme une image, soit de manière plus littérale, et de cette hésitation peut naître le fantastique. Dans un entretien, Harchi avoue ne pas souscrire au genre de la littérature « de banlieue » qui lui parait réducteur :

La question de la littérature de banlieue est très compliquée parce que ça met en œuvre à la fois l'ambition d'un individu à être reconnu comme un écrivain et, à la fois, les difficultés qui se posent à lui à partir du moment où il évoque un territoire particulier. Un roman de banlieue, c'est réducteur et c'est créer un sous-genre qui de manière formelle ou stylistique, n'existe pas. (Harchi 2009b)

13 Harchi soulève ici la question de l'étiquette littérature « de banlieue » qui en effet pose problème car mettre des romans dans cette catégorie implique une lecture souvent 
réductrice de textes qu'on ne lirait que comme des témoignages, appréciés non pas pour leurs qualités esthétiques mais plutôt pour ce qu'ils peuvent nous dire sur la vie en banlieue. Or Kaoutar Harchi propose un roman qui est lui-même un texte-limite, comme le définit Roland Barthes, c'est-à-dire non pas un texte qui se lit pour l'histoire mais qui s'écrit plutôt avec le lecteur (Barthes $1973: 23$ ) et qui ne s'inscrit pas tout à fait dans les problématiques contemporaines de la banlieue (discrimination, chômage, etc.), en tout cas pas de manière explicite. En conclusion, on peut dire que Kaoutar Harchi, qui est également sociologue, est particulièrement sensible au poids des traditions, et à l'intensité avec laquelle elles pèsent sur les personnages (thème récurrent dans ses autres romans) mais elle insiste également sur l'importance de l'environnement dans lequel évoluent ses personnages, la «zone cinglée » étant elle-même un personnage central du roman. Zone cinglée, qui s'inscrit dans la mouvance urbaine (dont la collection "Exprim' » aux éditions Sarbacane est spécialiste) donne lieu à des angles de saisie multiples, se lisant aussi bien comme un roman fantastique que comme une dystopie. Plutôt que d'alerter de manière explicite le lecteur sur les conditions de vie des habitants de banlieues défavorisées, l'auteure préfère jongler avec plusieurs genres, refusant d'ancrer le roman dans une époque particulière. Sur Nedjma, Harchi avait écrit: «les lieux de Nedjma s'apparentent à une vaste mosaïque, rebelle à toute forme de saisissement définitif » (Harchi 2012 : 118). L'influence de Kateb Yacine est claire dans ce premier opus qui échappe à la lecture parfois un peu réductrice de roman sur la banlieue. L'atmosphère apocalyptique de Zone Cinglée est transmise non seulement par sa forme donc, celle de la mosaïque, mais aussi par l'atmosphère d'une fiction qui finit littéralement en guerre civile, menant à la destruction de la zone.

\section{René : roman urbain d'anticipation?}

14 C'est justement dans cette atmosphère apocalyptique que s'ouvre le roman de Disiz, aussi connu sous le nom de Sérigne M. Gueye, auteur du livre Les Derniers de la rue Ponty paru en 2010, mais plus célèbre pour ses albums de rap. Cette esthétique du chaos caractérise donc le roman René, mais avec cette fois un ancrage dans le temps et dans un contexte politique bien précis : nous sommes en 2017 et Marine Le Pen est présidente. Le roman de Disiz s'ouvre sur une chronologie précise en guise de préface, qui retrace l'évolution du succès du Front national pour ensuite offrir un contexte post-émeutes :

Tout était parti de là, de cette verrue plantaire au nord de la ville. Lointaine circonscription du Grand Paris, le Havreux-sous-Boqueteau était devenu l'épicentre de la guerre civile. Il avait suffi d'un rien. Une énième bavure policière provoquée par quelques flics apeurés. Mais cette fois, c'était allé trop loin. Pour un simple contrôle d'identité, sept adolescents avaient trouvé la mort. (Disiz 2012 : 11)

Le roman s'apparente donc lui aussi à une dystopie, mais avec une chronologie claire et un contexte plus ancré dans les réalités sociales que celui d'Harchi, qui font plutôt de René un roman d'anticipation. Le nom de la ville de banlieue Havreux-sous-Boqueteau évoque Aulnay-sous-Bois ou Clichy-sous-Bois, lieux des émeutes de 2005 qui représentent dans le roman le point de départ d'une série de révoltes urbaines. Le personnage principal, René, évolue dans une société où le parti d'extrême droite a pris le pouvoir, et la France, dans un élan nationaliste, a quitté la zone euro pour mettre en circulation le franc européen (le FREU). Les noms des enfants d'origine étrangère ont dû être francisés. Ainsi on apprend que le vrai nom de René né d'une mère arabo-française et d'un père d'origine malienne est en fait Youcef, mais après la loi de 2013 de francisation des noms, sa mère avait choisi 
René, non pas comme le René de Chateaubriand, mais comme l'actrice qui jouait le rôle de Bridget dans Le Journal de Bridget Jones (Renée Zellweger). Une milice paramilitaire (le RAT, Répression Armée Territoriale) quadrille la banlieue, zone de non-droit rongée par la drogue, et les jeunes communiquent dans une langue simplifiée, proche de la langue des textos dans laquelle le QU n'existe plus (il est remplacé par le K). René est un adolescent timide, amoureux de littérature et en décalage par rapport aux avancées technologiques, à la recherche de ses origines et plus précisément de l'identité de son père, mort dans des circonstances inconnues et dont la mère refuse de révéler l'identité.

René est un roman futuriste qui montre ce que pourrait devenir la France si le Front national était au pouvoir. Le clivage Paris-banlieue s'est accru et l'esthétique du texte apparait comme étant étonnamment similaire à celle du film Banlieue 13, dans lequel la banlieue est totalement coupée de Paris, survivant de trafics en tous genres et dominée par les nouvelles technologies. Cette séparation mène à la création d'un contre-pouvoir d'où émergent des figures révolutionnaires. Dans Banlieue 13, des groupes de jeunes adultes d'origines diverses se liguent contre le gouvernement corrompu. Dans René, deux héros des banlieues, Balna et Abdoulaye (« le Che noir »), servent de figures héroïques à la jeunesse française issue de l'immigration, le roman laissant à croire que le Che noir pourrait être le père du héros. Bien que René puisse être considéré comme un roman d'anticipation plutôt réaliste, il flirte aussi avec le merveilleux. Par exemple, lorsque René a peur ou lorsqu'il est ému de manière générale, ses émotions prennent la forme d'une araignée ce qui n'est pas sans rappeler le nénuphar qui envahit le corps de Chloé dans L'Écume des jours de Boris Vian, auteur que lit René d'ailleurs :

Mais il avait peur de prendre une lame dans le foie devant la grille du collège, et cette peur, comme une araignée géante, déployait ses pattes sur son cœur et sa poitrine. Chaque jour, elle le poussait à se lever deux fois plus tôt afin de prendre un itinéraire plus sûr. (20)

Le roman de Disiz peut être qualifié de roman urbain pour les mêmes raisons que celui d'Harchi : couverture évoquant les grands ensembles, thèmes liés à l'urbanisme, clivage centre-banlieue, langue proche du langage des textos, etc. Toutefois, René est plus ancré dans les réalités socio-économiques et politiques d'aujourd'hui, ou de demain plutôt, puisque la langue du roman, empreinte de verlan et de langage «SMS » évoque le futur de par l'utilisation de l'InstinctPhone (19) qui normalise l'écriture phonétique, reléguant le français standard à une langue perçue comme érudite. Tout comme dans le roman Zen City, de Grégoire Hervier (2009), Disiz ne projette pas ses personnages dans un monde totalement futuriste, mais utilise des technologies existantes dont il exagère à peine les caractéristiques afin de rendre sa vision du futur plausible. À cet égard, la référence aux émeutes d'octobre et de novembre 2005 sert d'étape marquante dans la généalogie de la lutte de personnes souvent marginalisées et géographiquement isolées vivant dans la périphérie de la capitale.

D'un point de vue politique, Disiz met l'accent sur les inquiétudes des Français et inscrit le roman dans la lignée du livre Les Sauvages de Sabri Louatah (2011), ou de Soumission de Michel Houellebecq (2015), deux romans qui imaginent une France avec un président d'origine maghrébine. Ces deux romans indiquent un certain malaise quant au futur politique de la France, qui connaît une gauche divisée et une extrême droite montante, avec dans le cas de Soumission, un duel entre Marine le Pen et Mohammed Ben Abbes, le leader d'un parti fictif appelé « La Fraternité musulmane ». René évoque la possibilité d'un contre-pouvoir incarné par le Che noir, mais ce dernier est mort, ce qui symboliquement 
laisse envisager qu'il y a peu d'espoir en politique pour l'existence d'un contre-pouvoir. Le roman interroge donc l'avenir de la société française face aux troubles politiques et aux crispations identitaires. En effet, René donne au lecteur l'occasion de réfléchir sur l'identité française, comme l'indique un dialogue houleux entre Balna le leader et René dans lequel Balna refuse de voir un citoyen français, ce à quoi un journaliste témoin du dialogue répond: «C'est quoi une gueule de Français? Attends, français c'est pas une couleur » (95). Disiz, tout comme René, est métis, métissage qu'il avait déjà affirmé aux côtés de Yannick Noah dans une chanson justement intitulée Métis(se). Son positionnement est donc celui d'un Français aux origines multiples qui refuse les catégories et qui est culturellement ancré dans plusieurs univers. Le rappeur-écrivain interroge l'identité française, comme dans ses chansons où le personnage refuse d'être enfermé dans une catégorie spécifique, affirmant vouloir échapper aux clichés souvent associés aux jeunes de banlieue. Dans ce contexte, l'utilisation du mot ghetto n'est pas ici à prendre de manière littérale, ni comme abus de langage pour décrire les banlieues françaises (comme le rappelle Loïc Waquant dans Parias urbains, les banlieues en France ne sont pas des ghettos américains) mais plutôt comme une image faisant référence à une identité de «jeune de banlieue » faite de clichés, qui ne correspond pas à Disiz. Dans son morceau Complexité française, le rappeur affirme d'ailleurs :

Mon enfance en damier sur des dizaines de diapos

J'suis bien plus qu'un papier, bien plus qu'un drapeau

Si t'es certain de c'que t'es, j'te tire mon chapeau

J'me suis échappé de tous vos ghettos

Les références à une identité nationale (représentée par le drapeau ou les papiers d'identité) semblent être insuffisantes pour réellement dire ce qu'être Français au xxI siècle signifie. René se termine en tragédie, avec, tout de même l'espoir que le personnage principal, tout comme Tâarouk dans le roman d'Harchi, puisse quitter cette banlieue perdue. Comme dans Zone cinglée, dans lequel les Mères investissent la zone sud de la ville dont elles prennent le contrôle, on apprend vers la fin du roman qu'une génération de personnes aisées avait racheté une partie de la zone pour offrir à sa jeunesse une alternative au monde corrompu : «peu après les événements, la totalité de ces anciennes zones urbaines prioritaires avait été rachetée, mètre carré par mètre carré par toute une génération de quadragénaires avantagée par la vie » (170). Ces personnes recréèrent ainsi un peu de mixité sociale, permettant à des élèves issus de milieux défavorisés de côtoyer une population plus aisée, ce qui laisse à penser que la cohésion sociale passe par un urbanisme humain et la mixité sociale. La ville et le milieu urbain en général (incluant le périurbain ou la banlieue) seront probablement de plus en plus le lieu de dystopies au fur et à mesure que les technologies envahissent notre quotidien et modifient de manière durable les rapports entre habitants de la ville. C'est peut-être pour cette raison que certains urbanistes envisagent un retour vers la campagne dans un avenir proche où se mêlent souci de l'environnement et épanouissement personnel. C'est dans cette direction que va le travail du philosophe de l'urbain Thierry Pacquot :

En ce sens, l'utopie du $\mathrm{xxI}^{\mathrm{e}}$ siècle se souciera avant tout de l'écologie et de l'habitabilité de la demeure terrestre des hommes. L'éducation, les droits des enfants, la parité homme/femme, l'épanouissement sexuel, la réduction du temps de travail par la mécanisation des tâches, la possibilité de vivre selon ses propres rythmes et désirs, tout cela sera encore à l'ordre du jour, mais entièrement unifié par l'écotopie. (Paquot 2007 : 108) 
Les deux romans nous offrent donc des versions assez comparables de banlieues mises à feu, perçues comme des territoires perdus ou des zones de non-droit, avec une atmosphère post-apocalyptique. Zone cinglée et René contiennent des éléments permettant de qualifier ces textes de romans urbains, même si celui de Disiz s'approche plus du roman urbain typique de par sa langue et ses emprunts à la culture hip-hop. Le roman d'Harchi échappe à toute catégorie fixe et jongle entre allusions mythologiques et éléments de science-fiction, pour finalement nous donner un texte hybride pouvant se lire à travers plusieurs prismes. La vision dystopique de ces deux écrivains leur permet de mettre en garde le lecteur sur les dangers d'un urbanisme mal géré, avec la possibilité d'un état totalitaire pour Disiz et celle de la tension causée par un cloisonnement entre diverses zones pour Harchi. À la fin de Zone cinglée Tâarouk quitte la zone pour un voyage vers «l'autre pays ». Alors que la «zone cinglée » n'a pas de nom (même si l'on suppose qu'il s'agit de Paris), la destination finale du protagoniste est, elle, clairement mentionnée : Timimoun en Algérie, «le temps de retrouver l'autre langue» (187). Cette ouverture vers un autre espace à la fin du roman peut se lire de manière symbolique comme une impossibilité à trouver sa place dans une société qui tend vers la ségrégation sinon raciale en tout cas urbaine, creusant les écarts entre centre et périphérie. Dans René , la vie en périphérie est elle aussi synonyme de marginalisation sur plusieurs plans (économique, social, politique). Le roman de Disiz a d'ailleurs un caractère quasi prophétique, dans la mesure où certaines des mesures prises par le gouvernement décrites dans le roman (paru en 2012) ont été discutées en 2016, comme, la francisation des prénoms, du maire de Béziers Robert Ménard, qui souhaite une réforme de l'état civil pour donner une consonance plus « française» aux prénoms d'origine étrangère ${ }^{2}$. Que ce soit de manière explicite ou imagée, les deux romans en disent long sur les rapports entre l'urbain et l'individu, ainsi que sur les réalités sociales contemporaines. Grâce à l'utilisation de la dystopie, les deux écrivains créent des univers littéraires originaux et soulignent des problématiques actuelles en les exagérant à peine de manière à créer un futur plausible. De ce décalage minime entre présent et futur proche naît ainsi une inquiétude chez le lecteur qui est alors amené à réfléchir sur notre société actuelle.

\section{BIBLIOGRAPHIE}

Barthes, Roland, 1973, Le Plaisir du texte, Paris, Seuil.

Bornier, Évelyne, 2012, « Kaoutar Harchi : Zone cinglée, Apocalypse now made in banlieue », dans N. Redouane et Y. Bénayoun-Szmidt (dir.), Qu'en est-il de la littérature beur au féminin ?, Paris, L'Harmattan, p. 377-391.

Boule, Pierre, 1963, La Planète des singes, Paris, Julliard.

Céline, Louis-Ferdinand, 1932, Voyage au bout de la nuit, Paris, Denoël.

Disiz, 2012, René, Paris, Denoël.

Djaïdani, Rachid, 1999, Boumkoeur, Paris, Seuil.

Itinéraires, 2016-3 | 2017 
Harchi, Kaoutar, 2009a, Zone cinglée, Paris, Sarbacane.

Harchi, Kaoutar, 2009b, Entretien radio du 13 juillet, [En ligne], http://www.77info.fr/article-548Livres+d'+ete-Livres+d+ete+++zone+cinglee, consulté le 14 juin 2016.

Harchi, Kaoutar, 2012, « Entre exils, errances et migrations. L'expérience littéraire de Kateb Yacine ", Hommes \& Migrations, $\mathrm{n}^{\circ}$ 1298, p. 112-122, [En ligne], http://

hommesmigrations.revues.org/1584

DOI : 10.4000/hommesmigrations.1584

Hervier, Grégoire, 2009, Zen City, Vauvert, Au diable Vauvert.

Houellebeq, Michel, 2015, Soumission, Paris, Flammarion.

Louatah, Sabri, 2011, Les Sauvages, Paris, Flammarion.

Nothomb, Amélie, 2005, Acide sulfurique, Paris, Albin Michel.

Paquot, Thierry, 2007, Utopies et utopistes, Paris, La Découverte, coll. « Repères ».

Puig, Stève, 2011, «Littérature-monde et littérature urbaine : deux manifestes, même combat ? ", Nouvelles Francographies, vol. 2, n 1, p. 87-95.

Qui Fait la France?, 2007, Chroniques d'une société annoncée, Paris, Stock.

Stiénon, Valérie, 2012, « Dystopies de fin du monde. Une poétique littéraire du désastre », Culture, le magazine culturel de l'université de Liège, [En ligne], http://hdl.handle.net/2268/136116.

Todorov, Tzvetan, 1970, Introduction à la littérature fantastique, Paris, Seuil.

Vian, Boris, 1947, L'Écume des jours, Paris, Gallimard.

Wacquant, loïc, 2007, Parias urbains : ghetto, banlieues, État, Paris, La Découverte.

\section{NOTES}

1. «Entre 1830, date des premières anti-utopies constituées en récit, et 1950, moment de convergence de ces récits avec la science-fiction naissante, la production dystopique francophone s'avère riche, complexe et encore peu étudiée » (Stiénon $2012: 1$ ).

2. Voir l'article du journal Le Point du 26 mai 2016: http://www.lepoint.fr/politique/ presidentielle-2017-les-delirantes-propositions-de-robert-menard-26-05-2016-2042370_20.php (consulté le 12 janvier 2016).

\section{RÉSUMÉS}

Les rapports entre ville et banlieue sont au cœur de la littérature urbaine, mouvance littéraire apparue suite aux émeutes urbaines de 2005. Cette littérature aborde des thèmes variés dont la gestion de l'espace urbain et la vie quotidienne dans la périphérie de Paris. Deux romans récents Zone cinglée (2009) et René (2012) offrent une vision pessimiste de l'avenir des banlieues en insistant sur les dangers d'un urbanisme mal géré et sur les problèmes liés à la ségrégation urbaine qui est, dans les deux romans, poussée à l'extrême. La dystopie, genre qui se développe 
en littérature et au cinéma dans des contextes politiques souvent troubles, permet aux deux écrivains de faire un constat alarmant sur les conditions de vie dans certaines banlieues tout en alertant le lecteur quant à d'éventuels dérapages de la société française actuelle.

The relationship between the city and its outskirts are at the core of urban literature, a literary trend which appeared after the 2005 riots in France. This literature tackles various themes, including urban policies and daily life on the outskirts of Paris. Two recently published novels, Zone cinglée (2009) and René (2012), offer a rather pessimistic vision of the future of the French peripheries by focusing on failed urban policies and problems linked to urban segregation which are prevailing in both novels. Dystopia, a popular genre in literature and film in times of political trouble, allows the two authors to draw an alarming portrait of life in the banlieues while warning the reader about potential dangers in contemporary French society.

\section{INDEX}

Mots-clés : littérature urbaine, banlieue, dystopie, hip-hop, urbanisme, Kaoutar Harchi, Disiz

Keywords : urban literature, ghetto, dystopia, hip-hop, urbanism, Kaoutar Harchi, Disiz

\section{AUTEUR}

\section{STÈVE PUIG}

St John's University, New York, USA 